\title{
Colorectal surgery during the COVID-19 outbreak: do we need to change?
}

\author{
Marco Ettore Allaix ${ }^{1}\left(\mathbb{D} \cdot\right.$ Giacomo Lo Secco $^{1} \cdot$ Francesco Velluti $^{2} \cdot$ Paolo De Paolis ${ }^{2} \cdot$ Simone Arolfo ${ }^{1} \cdot$ Mario Morino $^{1}$
}

Received: 5 July 2020 / Accepted: 8 December 2020 / Published online: 2 January 2021

(c) Springer Nature Switzerland AG part of Springer Nature 2021

\begin{abstract}
The outbreak of COVID-19 has led some leading surgical societies to postpone colorectal cancer resections, support the employment of low-risk strategies in patients requiring colorectal surgery, such as construction of a stoma rather than primary anastomosis, in order to minimize the risk of potentially life-threatening complications. They have also recommended against the use of the laparoscopic approach. However, the evidence supporting these recommendations is scarce. The aim of this study was to assess the outcomes of colorectal resections during the COVID-19 pandemic. This is a retrospective review of a prospective institutional database. All consecutive patients undergoing elective or emergent colorectal resections between March 9 and April 15, 2020, were compared to those treated in the same period of time in 2019. Despite an overall reduction in the surgical activity of $56.3 \%$ in 2020, the two groups were similar in terms of absolute numbers of colorectal resections, type of surgery and use of laparoscopy. The overall postoperative complications rate was similar: $20 \%$ in 2019 versus $14.9 \%$ in $2020(p=0.518)$, without any difference in terms of severity. No patient during the postoperative course got infected by COVID-19, as well as none from the surgical team. Median length of hospital stay was 5 days in both groups $(p=0.555)$. Postponing surgery in colorectal cancer patients and performing more stomas rather than direct anastomosis is not justified. The routine use of laparoscopy should not be abandoned, thus not depriving patients of its clinically relevant early short-term benefits over open surgery.
\end{abstract}

Keywords COVID-19 $\cdot$ Colorectal $\cdot$ Laparoscopy $\cdot$ Anastomosis $\cdot$ Morbidity $\cdot$ Recommendations

\section{Introduction}

The outbreak of coronavirus-19 (COVID-19) has been stretching the healthcare systems worldwide since 3 months: The virus has spread very quickly, affecting a large number of individuals with subsequent need for hospitalization and, in most severe cases, treatment in intensive care units (ICUs). All around the world, governing bodies had to face this unprecedented scenario, reorganizing the healthcare services and reallocating resources. Rationing and prioritising of surgical interventions have rapidly become the goals of the governments that allowed only urgent/emergent and oncologic surgeries in most countries.

Marco Ettore Allaix

marcoettore.allaix@unito.it

1 Department of Surgical Sciences, University of Torino, Torino, Italy

2 Department of Surgery, Città della Salute e della Scienza Hospital, Torino, Italy
Parallel to these political decisions, most major surgical societies have released recommendations aiming at guiding surgeons in their daily decision-making process for prioritization of surgery in the emergent setting and in cancer patients [1-6]. Some of these guidelines recommend a shift toward a non-operative treatment strategy for acute abdominal conditions, such as acute appendicitis or cholecystitis. They also recommend against the use of the laparoscopic approach, due to the potential increased risk of intraperitoneal virus aerosolization and higher exposure risk for the operating room staff.

Based on some early data suggesting that cancer patients are at higher risk of COVID-19-related infection, severe course and mortality $[7,8]$, it has been advised to postpone resection for colorectal cancer within 3 months after the diagnosis. In addition, some societies support the employment of low-risk strategies, such as construction of a stoma rather than primary anastomosis in order to minimize the risk of potentially life-threatening complications, such as anastomotic leak. However, the evidence supporting 
these recommendations is scant. We believe that the existing knowledge is insufficient to radically change the best established surgical practice. Therefore, at the Department of Surgery of our Institution, we have not changed surgical techniques and approaches during the COVID-19 outbreak.

The aim of this study was to assess the outcomes of colorectal resections performed during the COVID-19 pandemic in a tertiary referral academic center and to compare the clinical outcomes with those obtained in patients undergoing colorectal surgery in the same period of time in 2019.

\section{Materials and methods}

On March 8 2020, a National Decree has forced the Italian surgical Units to limit their activity to urgency/emergency and cancer surgery. All consecutive patients undergoing elective or emergent colorectal resections at the Department of Surgery, University of Torino, Italy, between March 9 and April 15, 2020, were included in the study. Patients' characteristics, type of surgery, laparoscopic or open approach, short-term complication rate ( $<30$ days), anastomosis leak rate and length of hospital stay were collected and compared with the records of all consecutive patients who underwent colorectal resections in the same period of the year before. The patients who entered the emergency department (ED) with an acute condition followed two totally separate pathways, depending on the presence or absence of any symptom possibly related to the COVID-19. All patients with indication for surgery were then screened for COVID-19 preoperatively in $\mathrm{ED}$, even if asymptomatic. In addition, all patients scheduled for elective surgery were tested prior to surgery. If negative, they underwent the planned surgery, otherwise the operation was postponed and rescheduled after resolution of COVID-19 infection. Healthcare personnel was regularly screened for COVID-19 by a nasopharyngeal swab. A smoke filtration system was used, and pneumoperitoneum was safely evacuated in all laparoscopic procedures. During postoperative course, patients who developed cough, fever or respiratory symptoms were newly tested for COVID-19 and, if positive, moved to a COVID area.

\section{Statistical analyses}

Continuous variables are reported as median and range, while categorical variables as events number and percentages. Differences between 2019 and 2020 operative data were tested with the Fisher's exact test for categorical variables and with the Mann-Whitney test for continuous ones. All $p$ values were two-sided. A level of $5 \%$ was set as the criterion for statistical significance. The statistical analysis was performed using SPSS version 19 (Copyright @ SPSS Inc., 2000).

\section{Results}

Despite an overall reduction in the general surgery activity of $56.3 \%$ in 2020 (163 procedures in 2020 vs. 373 procedures in 2019) as a consequence of the restrictions imposed to surgical activity in Italy, the absolute number of colorectal resections was similar in the two study periods: 75 in 2019 and 74 in 2020.

Patients' characteristics are summarized in Table 1.

A patient without COVID-19-related symptoms was diagnosed with COVID-19 infection at the preoperative screening: The operation was delayed after obtaining a double check of swab negativity at 15 days. No postoperative complications were recorded.

The rate of resections for cancer slightly increased in 2020 (32 [42.6\%] in 2019 vs. 44 [59.5\%] in 2020; $p=0.049)$, and laparoscopic approach was adopted in a similar percentage of cases $(72.0 \%$ in 2019 vs. $81.1 \%$ in $2020 ; p=0.247)$. Table 1 shows that similar rates of right/ left colectomies, sigmoidectomies and anterior resections were performed in 2019 and 2020. The overall number of colorectal resections with direct anastomosis was $46 / 75$ $(61.3 \%)$ in 2019 and $48 / 74(64.9 \%)$ in 2020 , with an anastomotic leak rate of $10.9 \%$ in 2019 vs $4.2 \%$ in 2020 $(p=0.263)$. The overall postoperative complications rate was similar: $20 \%$ in 2019 vs $14.9 \%$ in $2020(p=0.518)$, without any difference in terms of severity according to Clavien-Dindo classification [9]. Pathological data were comparable between the two groups, without any significant difference (Table 2).

No patient during the postoperative course got infected by COVID-19, as well as none from the surgical team.

The median length of postoperative hospital stay was 5 days in both groups $(\mathrm{p}=0.555)$.

\section{Discussion}

The COVID-19 pandemic has dramatically challenged the national health systems worldwide during the last few months. It became shortly obvious that saving and reallocating resources was mandatory to face this unprecedented emergency. A quick change in the surgical priorities was deemed crucial by the governing bodies all around the world in order to limit the risk of exposure to COVID-19 of both healthcare professionals and patients.

Parallel to the governments, most surgical societies, including the American College of Surgeons (ACS) [1], 
Table 1 Colorectal resections: comparison between 2019 and 2020

\begin{tabular}{|c|c|c|c|c|c|}
\hline & 2019 & & 2020 & & $p$ value \\
\hline Operations $(n ; \%)$ & $75(100)$ & & $74(100)$ & & \\
\hline Resections for cancer $(n ; \%)$ & 32 & $42.6 \%$ & 44 & $59.5 \%$ & $0.049^{\mathbb{I}}$ \\
\hline Age (years) & $69(16-93)$ & & $67(18-89)$ & & $0.889^{\#}$ \\
\hline Medical comorbidities $(n ; \%)$ & 34 & $45.3 \%$ & 41 & $55.4 \%$ & 0.253 \\
\hline Cardiovascular & 17 & $22.6 \%$ & 16 & $21.6 \%$ & $1^{\text {II }}$ \\
\hline Pulmonary & 6 & $8.0 \%$ & 7 & $9.5 \%$ & $0.780^{\mathbb{I I}}$ \\
\hline Renal & 3 & $4.0 \%$ & 10 & $9.5 \%$ & $0.046^{\mathbb{I}}$ \\
\hline Diabetes & 6 & $8.0 \%$ & 1 & $13.5 \%$ & $0.116^{\mathbb{I}}$ \\
\hline Cirrhosis & 2 & $2.7 \%$ & 1 & $1.3 \%$ & $1^{\mathbb{I I}}$ \\
\hline Laparoscopy $(n ; \%)$ & 54 & $72.0 \%$ & 60 & $81.1 \%$ & $0.247^{\mathbb{I}}$ \\
\hline Conversions $(n ; \%)$ & $6 / 54$ & $11.1 \%$ & $2 / 60$ & $3.3 \%$ & $0.147^{\mathbb{I}}$ \\
\hline \multicolumn{6}{|l|}{ Type of resection } \\
\hline Right hemicolectomy & 15 & $20.0 \%$ & 20 & $27.0 \%$ & 0.339 \\
\hline Left hemicolectomy & 9 & $12.0 \%$ & 8 & $10.8 \%$ & $1^{\mathbb{T}}$ \\
\hline Sigmoidectomy & 6 & $8.0 \%$ & 3 & $4.1 \%$ & $0.494^{\mathbb{I}}$ \\
\hline Anterior resection & 2 & $2.7 \%$ & 10 & $13.5 \%$ & $0.017^{\mathbb{I}}$ \\
\hline Hartmann procedure & 2 & $2.7 \%$ & 1 & $1.3 \%$ & $1^{\text {II }}$ \\
\hline Transanal endoscopic microsurgery & 7 & $9.3 \%$ & 4 & $5.4 \%$ & $0.533^{\mathbb{I}}$ \\
\hline Appendectomy & 12 & $16 \%$ & 11 & $14.9 \%$ & $1^{\text {II }}$ \\
\hline Others & 22 & $29.3 \%$ & 17 & $23.0 \%$ & $0.457^{\mathbb{I}}$ \\
\hline Complications $(n ; \%)$ & 15 & $20.0 \%$ & 11 & $14.9 \%$ & $0.518^{\mathbb{I}}$ \\
\hline Dindo 1-2 & 9 & $12.0 \%$ & 6 & $8.2 \%$ & $0.588^{\mathbb{I}}$ \\
\hline Dindo 3 & 4 & $5.3 \%$ & 2 & $2.7 \%$ & $0.681^{\mathbb{I}}$ \\
\hline Dindo 4-5 & 2 & $2.7 \%$ & 3 & $4.0 \%$ & $0.681^{\mathbb{I}}$ \\
\hline Anastomotic leak $(n ; \%)$ & $5 / 46$ & $10.9 \%$ & $2 / 48$ & $4.2 \%$ & $0.263^{\mathbb{I}}$ \\
\hline
\end{tabular}

Table 2 Pathologic characteristics: comparison between 2019 and 2020

\begin{tabular}{llllll}
\hline & 2019 & \multicolumn{5}{c}{2020} & $p$ value \\
\hline Resection for cancer & $32 / 75$ & $42.6 \%$ & $44 / 74$ & $54.5 \%$ & $0.049^{\mathbb{I}}$ \\
$T$ stage & & & & & \\
High-grade dysplasia & 3 & $9.4 \%$ & 8 & $18.2 \%$ & $0.339^{\mathbb{I}}$ \\
Tis & 5 & $15.5 \%$ & 3 & $6.8 \%$ & $0.269^{\mathbb{I}}$ \\
1 & 0 & $0 \%$ & 1 & $2.3 \%$ & $1^{\mathbb{I}}$ \\
2 & 6 & $18.8 \%$ & 7 & $15.9 \%$ & $0.766^{\mathbb{I}}$ \\
3 & 12 & $37.5 \%$ & 18 & $40.9 \%$ & $0.815^{\mathbb{I}}$ \\
4 & 6 & $18.8 \%$ & 7 & $15.9 \%$ & $0.766^{\mathbb{I}}$ \\
$N$ stage & & & & & \\
Nx & 7 & $21.9 \%$ & 8 & $18.2 \%$ & $0.774^{\mathbb{I}}$ \\
0 & 19 & $59.4 \%$ & 17 & $38.6 \%$ & $0.104^{\mathbb{I}}$ \\
1 & 5 & $15.6 \%$ & 11 & $25.0 \%$ & $0.399^{\mathbb{I}}$ \\
2 & 1 & $3.1 \%$ & 8 & $18.2 \%$ & $0.071^{\mathbb{I}}$ \\
Resection margin & & & & & $0.421^{\mathbb{I}}$ \\
0 & 31 & $96.9 \%$ & 44 & $100.0 \%$ & \\
1 & 1 & $3.1 \%$ & 0 & $0 \%$ & \\
Tumor diameter (cm) & 4 & $1.8-10$ & 3.75 & $1-7.5$ & $0.238^{\#}$ \\
Number of lymph & 16 & $3-32$ & 19 & $6-64$ & $0.610^{\#}$ \\
nodes harvested & & & & & \\
\hline
\end{tabular}

IIFisher's exact test; \# Mann-Whitney test the Society of the American Gastrointestinal and Endoscopic Surgeons (SAGES) [2], the European Association for Endoscopic Surgery (EAES) [2], and the Royal College of Surgeons (RCS) [3], have released recommendations to help surgeons in their decision-making processes. Early reports suggested that development of COVID-19 infection during the early postoperative course is associated with high morbidity and mortality [10]. Therefore, urgent life-threatening conditions and cancers have been prioritized, while elective surgery for most benign diseases has been postponed after the end of the pandemic. Main factors to be considered when prioritizing surgery and defining surgical timing are clinical presentation, tumor stage, type of surgical procedure, patient's conditions and the crisis phase at the index hospital (phase 1 vs. phase 2 vs. phase 3 ).

The management of colorectal cancer patients during COVID-19 pandemic represents one of the main current concerns, with several areas of uncertainty and controversies $[11,12]$. Since some recent data suggest that cancer patients are at higher risk of COVID-19 infection and more likely adverse outcomes if infected [7, 8], a discussion has been raised worldwide about the opportunity to modify cancer treatment protocols (use of neoadjuvant or 
adjuvant treatments, timing for surgery, etc.). Some societies have advised to consider low-risk procedures in order to minimize the probability of developing complications requiring postoperative recovery in ICU (i.e., stoma vs. direct colorectal anastomosis); non-operative management options in selected patients have also been proposed.

To date, possible implications of the changes proposed to cancer management pathways during the COVID-19 pandemic on the prognosis of cancer patients are poorly understood. The concept of postponing surgery after the pandemic or using other treatment modalities is challenged by inconsistent results of the studies published before COVID-19 pandemic assessing survival according to different ranges of delay in surgical resection [13-16]. Furthermore, the end of COVID-19 pandemic is far from being defined. While this policy might help reallocate resources, it might jeopardize patients' survival and expose surgeons, oncologists and radiotherapists to legal issues in the future once this crisis is over, in case of adverse oncologic outcomes.

The only study that has evaluated the short-term impact on surgical oncology of the aforementioned changes is a survey conducted by the COVID-SURGE-ITA group in Italy, the first country hit by COVID-19 in the Western world [17]. The survey was sent to 54 referral centers for HPB, colorectal, esophago-gastric and sarcoma/soft tissue cancers. A total of 11 colorectal units were involved. At 5 weeks, after the first case of secondary COVID-19 transmission in Italy, 82\% of colorectal units reported a reduction in surgical activity (median, -60\%). Alternative treatment to surgery was prioritized in $36 \%$ of centers.

To our best knowledge, ours is the first study that evaluates the clinical outcomes in a series of patients undergoing colorectal surgery during COVID-19 pandemic and challenges current surgical recommendations. The study has been conducted in Torino (Piedmont, Italy) at the biggest academic tertiary referral center of Northern Italy. Currently, Piedmont is one of the most affected areas in Italy, with 29,070 COVID-19 cases and 3,460 deaths related to COVID-19 by May 14, 2020 [18]. Since the beginning of COVID-19 outbreak, our hospital has went through phase 1 and phase 2 , and now is slowly going back to phase 1 . Following the Italian national guidelines, only emergent/urgent cases and oncologic patients were surgically treated at our hospital during the study period.

We believe that, since little is known about COVID-19, existing data are insufficient to radically change the best established approaches to patients with colorectal cancer. Therefore, we have not modified surgical techniques and approaches during the COVID-19 outbreak at our Institution. In particular, the types of procedures did not change during the COVID-19 outbreak, and a stoma rather than a direct anastomosis was not routinely fashioned after colorectal resection in the absence of a specific indication; the data analysis did not show significant differences in anastomotic leak rates when the two groups of patients were compared. Furthermore, we did not postpone nor restricted the indications for surgery in colorectal cancer patients.

The use of the laparoscopic approach is one of the most controversial topics during the COVID era [19-21]. While the laparoscopic approach to many abdominal diseases has well-known advantages over open surgery, the Intercollegiate General Surgery Guidance in the UK has suggested to avoid laparoscopy, advising to consider laparoscopy only in selected cases during the COVID-19 endemic. This statement is based on the hypothesis of increased risk of virus aerosolization when electrosurgical devices are used. Even though there is no evidence of COVID-19 emission and transmission during laparoscopic surgery, this suggestion has been quickly shared by surgeons worldwide via the social networks and the use of laparoscopy banned in several areas, thus potentially depriving patients from the major advantages of the minimally invasive approach. In the absence of evidence of COVID-19 in surgical smoke, we kept performing laparoscopy following the rules of good surgical practice established almost 25 years ago, such as avoiding desufflation of the abdomen without a smoke evacuation system to reduce postoperative pain, minimize the risk of tumor cell seeding at the trocar sites in oncologic patients $[22,23]$ and, at the same time, protect from potential virus spreading. During the postoperative course, no patient nor OR staff was infected by COVID-19.

We acknowledge that this study has some limitations. First, it is a single-Institution study, and therefore the results observed might not be generalized; second, the number of patients included is limited. However, we feel that it contributes to shed more light on some clinically relevant issues: 1) Morbidity and mortality are not increased during the COVID-19 outbreak, provided that a COVID-19-protected pathway is guaranteed to the surgical patients; therefore, postponing surgery in colorectal cancer is unjustified;2) there is no increased anastomotic leak rate; thus, performing more stomas rather than direct anastomosis is not justified, also considering that both stomas and stoma reversal have significant morbidity; and 3) the routine use of laparoscopy should not be abandoned, thus not depriving patients of its clinically relevant early short-term benefits over open surgery.

\section{Conclusions}

We are facing a unique period of uncertainty and fear, since this novel pandemic is challenging our traditional medical and surgical treatment options. Since COVID-19 is highly virulent and is associated with high morbidity and mortality, prompt answers to many questions regarding our patients' 
management are required. However, our clinical practice should be always driven by the evidence, that is currently lacking. Therefore, a call for high-quality research within international collaboration is mandatory to better define if we really need to modify our surgical indications and surgical approaches.

Author contributions MEA, PDP, SA and MM made substantial contributions to the conception and design of the study. GLS, FV, and SA collected the data and performed the analysis. MEA, PDP and MM participated in the interpretation of the data. MEA, SA and MM drafted the manuscript. All authors revised the draft critically and contributed to the final version of the manuscript. All authors read the final manuscript and approved the revised final version. All authors agree to be accountable for all aspects of the work in ensuring that questions related to the accuracy or integrity of any part of the work are appropriately investigated and resolved.

Funding No sources of support.

Data availability Datasets are available upon editors' request.

\section{Compliance with ethical standards}

Conflicts of interest The authors declare that they have no conflict of interest.

Ethical approval All procedures performed involving human participants were in accordance with the ethical standards of the institutional and/or national research committee and with the 1964 Declaration of Helsinki and its later amendments or comparable ethical standards.

Informed Consent No informed consent was required for this study.

\section{References}

1. https://www.facs.org/covid-19/clinical-guidance/elective-case/ colorectal-cancer

2. Francis N, Dort J, Cho E, Feldman L, Keller D, Lim R, Mikami D, Phillips E, Spaniolas K, Tsuda S, Wasco K, Arulampalam T, Sheraz M, Morales S, Pietrabissa A, Asbun H, Pryor A (2020) SAGES and EAES recommendations for minimally invasive surgery during COVID-19 pandemic. Surg Endosc. 3(6):2327-2331. https://doi.org/10.1007/s00464-020-07565-w

3. https://www.england.nhs.uk/coronavirus/wp-content/uploads/sites /52/2020/03/C0221-specialty-guide-surgical-prioritisation-v1.pdf

4. https://www.acpgbi.org.uk/content/uploads/2020/03/ACPGB I-statement-on-CRC-treatment-during-COVID-19-FINAL.pdf

5. https://www.esmo.org/guidelines/cancer-patient-management -during-the-covid-19-pandemic/gastrointestinal-cancers-color ectal-cancer-crc-in-the-covid-19-era

6. https://www.nccn.org/covid-19/pdf/Colorectal\%20COVID-19.pdf

7. Liang W, Guan W, Chen R, Wang W, Li J, Xu K, Li C, Ai Q, Lu W, Liang H, Li S, He J (2020) Cancer patients in SARS-CoV-2 infection: a nationwide analysis in China. Lancet Oncol 21:335-337

8. Wang H, Zhang L (2020) Risk of COVID-19 for patients with cancer. Lancet Oncol 21:e181

9. Dindo D, Demartines N, Clavien PA (2004) Classification of surgical complications: a new proposal with evaluation in a cohort of 6,336 patients and results of a survey. Ann Surg 240:205-213
10. Aminian A, Safari S, Razeghian-Jahromi A, Ghorbani M, Delaney CP (2020) COVID-19 outbreak and surgical practice: unexpected fatality in perioperative period. Ann Surg. Mar. 272(1):e27-e29

11. Ueda M, Martins R, Hendrie PC, McDonnell T, Crews JR, Wong TL, McCreery B, Jagels B, Crane A, Byrd DR, Pergam SA, Davidson NE, Liu C, Stewart FM (2020) Managing cancer care during the COVID-19 pandemic: agility and collaboration toward a common goal. J Natl Compr Canc Netw 18(4):366-369. https:// doi.org/10.6004/jncen.2020.7560

12. Al-Shamsi HO, Alhazzani W, Alhuraiji A, Coomes EA, Chemaly RF, Almuhanna M, Wolff RA, Ibrahim NK, Chua MLK, Hotte SJ, Meyers BM, Elfiki T, Curigliano G, Eng C, Grothey A, Xie C (2020) A practical approach to the management of cancer patients during the novel Coronavirus disease 2019 (COVID19) pandemic: an international collaborative group. Oncologist 25:e936-945

13. Wanis KN, Patel SVB, Brackstone M (2017) Do moderate surgical treatment delays influence survival in colon cancer? Dis Colon Rectum 60(12):1241-1249

14. Amri R, Bordeianou LG, Sylla P, Berger DL (2014) Treatment delay in surgically-treated colon cancer: does it affect outcomes? Ann Surg Oncol 21:3909-3916

15. Grass F, Behm KT, Duchalais E, Crippa J, Spears GM, Harmsen WS, Hübner M, Mathis KL, Kelley SR, Pemberton JH, Dozois EJ, Larson DW (2020) Impact of delay to surgery on survival in stage I-III colon cancer. Eur J Surg Oncol 46(3):455-461

16. O'Leary MP, Choong KC, Thornblade LW, Fakih MG, Fong Y, Kaiser AM (2020) Management considerations for the surgical treatment of colorectal cancer during the global Covid-19 pandemic. Ann Surg. 272(2):e98-e105. https://doi.org/10.1097/ SLA.0000000000004029

17. Torzilli G, Viganò L, Galvanin J, Castoro C, Quagliuolo V, Spinelli A, Zerbi A, Donadon M, Montorsi M, COVID-SURGE-ITA group, (2020) A snapshot of elective oncological surgery in Italy during COVID-19 emergency: pearls, pitfalls and perspectives. Ann Surg. 272(2):e112-e117. https://doi.org/10.1097/SLA.00000 00000004081

18. http://www.salute.gov.it/portale/nuovocoronavirus/dettaglioC ontenutiNuovoCoronavirus.jsp?id=5351\&area=nuovoCoronaviru s\&menu=vuoto

19. Zheng MH, Boni K, Fingerhut A (2020) minimally invasive surgery and the novel coronavirus outbreak: lessons learned in China and Italy. Ann Surg. 272(1):e5-e6. https://doi.org/10.1097/ SLA.0000000000003924

20. Vigneswaran Y, Prachand VN, Posner MC, Matthews JB, Hussain M (2020) What is the appropriate use of laparoscopy over open procedures in the current COVID-19 climate? J Gastrointest Surg. 24(7):1686-1691. https://doi.org/10.1007/s11605-020-04592-9

21. Chadi SA, Guidolin K, Caycedo-Marulanda SA, Spinelli A, Quereshy FA, Okrainec A (2020) Current evidence for minimally invasive surgery during the COVID-19 pandemic and risk mitigation strategies: a narrative review. Ann Surg. 272(2):e118-e124. https://doi.org/10.1097/SLA.0000000000004010

22. Tseng LN, Berends FJ, Wittich P, Bouvy ND, Marquet RL, Kazemier G, Bonier HJ (1998) Port-site metastases. Impact of local tissue trauma and gas leakage. Surg Endosc 12:1377-1380

23. Brundell SM, Tucker K, Texler M, Brown B, Chatterton B, Hewett PJ (2002) Variables in the spread of tumor cells to trocars and port sites during operative laparoscopy. Surg Endosc 16:1413-1419. https://doi.org/10.1007/s00464-001-9112-8

Publisher's Note Springer Nature remains neutral with regard to jurisdictional claims in published maps and institutional affiliations. 